Conf or nat i onal changes i $n$ reconst i t ut ed skel et al miscl e thi $\mathrm{n}$ fil ament s observed by fI uor escence spect roscopy

\begin{tabular}{|l|l|}
\hline 著者 & M K Nasao \\
\hline $\begin{array}{l}\text { j our nal or } \\
\text { publ i cat i on t i t l e }\end{array}$ & Advances i n Exper i ment al Medi ci ne and Bi ol ogy \\
\hline vol une & 592 \\
\hline page range & $111-123$ \\
\hline year & 2007 \\
\hline URL & ht t p: //hdl . handl e. net /10098/1768 \\
\hline
\end{tabular}




\title{
CONFORMATIONAL CHANGES IN RECONSTITUTED SKELETAL MUSCLE THIN FILAMENTS OBSERVED BY FLUORESCENCE
}

\author{
Masao $\mathrm{MiKi}^{1}$
}

\section{INTRODUCTION}

The cyclic interaction of myosin and actin coupled ATP hydrolysis generates the mechanical force of muscle contraction. During this process, the system passes through several steps. One of these is thought to be identical to the stable rigor complex formed by myosin and actin in the absence of ATP. This cyclic interaction is regulated by changes in tropomyosin ( $\mathrm{Tm})$ and troponin (Tn) located on the actin filament in response alterations in intracellular $\mathrm{Ca}^{2+}$ concentration (Ebashi et al., 1969). Tm contains seven quasi-equivalent regions, each of which has a pair of putative actin-binding motifs. Tn comprises three different subunits, TnC, TnI, and TnT. TnI alone inhibits actomyosin ATPase activity which is removed on adding $\mathrm{TnC}$, irrespective of $\mathrm{Ca}^{2+}$ concentration. TnT is required for full $\mathrm{Ca}^{2+}$-regulation of the ATPase activity of a reconstituted system (Ohtsuki et al., 1986). The globular part of the Tn complex (TnC, TnI and the C-terminal region of TnT) is located on residues 150-180 of Tm (White et al., 1987), and the elongated part, composed of the $\mathrm{N}$-terminal region, covers an extensive region of the $\mathrm{C}$-terminal half of $\mathrm{Tm}$. The binding of $\mathrm{Ca}^{2+}$ to $\mathrm{TnC}$ induces a series of conformational changes in the other components of the thin filament. This allows the effective association of myosin with actin, thus producing force. Although numerous studies have characterized the interaction between these thin filament proteins, the molecular mechanism whereby the $\mathrm{Ca}^{2+}$-trigger is propagated from $\mathrm{TnC}$ to the rest of the thin filament is still not well understood.

$\mathrm{X}$-ray diffraction studies of muscle fibers showed a significant change in the layer line intensities depending on whether the muscle was in a relaxed or contracted state (Huxley 1972; Haselgrove 1972). These intensity changes were interpreted as an azimuthal shift of the Tm strands in the grooves of the actin helix. Thus, the steric blocking theory was proposed in which Tm physically blocks the myosin-binding site on actin during relaxation. However, instead of a two-state model based on $\mathrm{Ca}^{2+}$-induced

\footnotetext{
1 Department of Applied Chemistry and Biotechnology, Research and Education Program for Life Science, Fukui University, Fukui 910-8507 Japan, TEL: +61-776-27-8786 FAX: +61-776-27-8747, e-mail masao@acbio2.acbio.fukui-u.ac.jp
} 
on-off switching, kinetic measurements have proposed a three-state model (McKillop \& Geeves, 1993). In this model, thin filaments exist in rapid equilibrium between three states (blocked or relaxed, closed, and open). The equilibrium between the relaxed and closed states is calcium-sensitive, and strong myosin binding stabilizes the open state. Recent X-ray diffraction and three-dimensional image reconstructions of electron micrographs (3D-EM) have led to a new model in which Tm moves between three distinct states (Holmes, 1995; Vibert et al., 1997). However, the $\mathrm{Ca}^{2+}$-induced conformational change of the thin filament clearly cannot be assigned to the movement of $\mathrm{Tm}$, since this interpretation assumes that the mass of the $\mathrm{Tn} / \mathrm{Tm}$ complex is distributed evenly and smoothly along the continuous Tm strands. Squire and Morris (1998) suggested that conformational changes observed from X-ray diffraction (and 3D-EM) data could be explained as movement of Tn instead of Tm. Narita et al. (2001) reconstructed three-dimensional images of thin filaments from cryo-electron micrographs without imposing helical symmetry and showed that the major change due to $\mathrm{Ca}^{2+}$ is a shift of Tn head (the main body of Tn). They suggested that while the N-terminal half of Tm does not undergo a major shift, the C-terminal one-third of Tm and/or Tn tail shifts significantly.

Fluorescence resonance energy transfer (FRET) has been used as a spectroscopic ruler in the range of several tens angstroms (Stryer, 1978). This method is especially valuable for detecting small structural changes (several $\AA$ ), because the FRET efficiency is a function of the inverse sixth power of the distance between probes. Furthermore, fluorescence donor and acceptor molecules can be specifically attached to proteins thus assigning a vector to the conformational change. Here we use FRET to detect conformational changes in skeletal muscle thin filament proteins in both relaxed and active states, and we review the recent research on the structural changes of the skeletal muscle thin filament induced by $\mathrm{Ca}^{2+}$ - and/or myosin-binding.

\section{STRUCTURAL CHANGES IN THIN FILAMENTS MEASURED BY FRET}

FRET measurements require that the energy donor and acceptor probes be attached to specific positions on proteins. The requirement for specific labeling limits the application of FRET method. However, recent progress in genetic engineering facilitated the selective labeling of protein and made it easier to introduce reactive amino acid side chains by introducing single cysteine at the required locations in proteins.

\section{2-1 Probe Sites on Actin, Tm, and Tn}

Actin was labeled with an energy acceptor probe at: (1) Gln-41 using fluorescein cadaverine (FLC); (2) Lys-61 using fluorescein 5-isothiocyanate (FITC); (3) Cys-374 using 4-dimethylaminophenyl 4'-maleimide (DABMI); or (4) the nucleotide-binding site using 2'(or 3')-O-(2,4,6-trinitrophenyl) adenosine 5'-diphosphate (TNP-ADP). Figure 1 A shows the locations of these labeling sites on a cross section of the atomic model of F-actin. Single cysteine $\alpha \mathrm{Tm}$ mutants were expressed in Escherichia coli in which additional residues A-A-S- or A-S- were added at the N-terminus in order to restore their binding ability to F-actin (Monteiro et al., 1994). These $\alpha$ Tm mutants (Cys-87, 237, 245, 
247 or 252) and native $\alpha \operatorname{Tm}$ (Cys-190) were labeled with the energy donor probe, 5-(2-iodoacetylaminoethyl)-amnonaphtalene-1-sulfonic acid (IAEDANS). Single cysteine mutants of TnI and TnT25k were also expressed in E. coli. TnT25k, the 25-kDa fragment (residues: 59-266) of TnT, is functionally identical to native TnT and is soluble at low ionic strength, unlike the native TnT. These TnI mutants (Cys-1, 9, or 181), native TnI (Cys-133) and TnT25k (Cys-60 or 250) were labeled with the energy donor probe, IAEDANS. The labeled Tn subunits were incorporated into the Tn complex with other unlabeled subunits prepared from rabbit skeletal muscle. Figure 1B shows the locations of the FRET energy donor probes on a schematic model of the Tm-Tn complex.
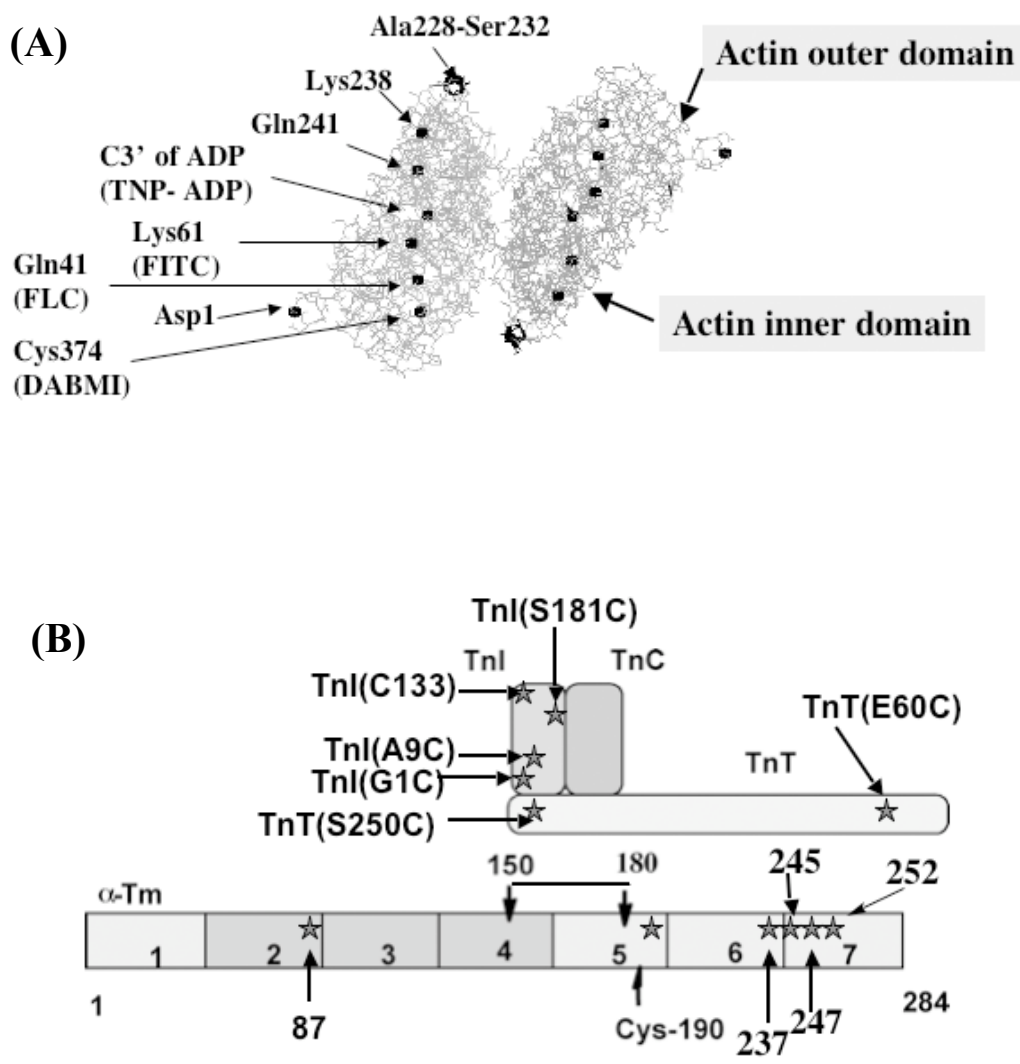

Figure 1: Locations of energy acceptor- and donor-labeling sites on a cross-section of an atomic model of F-actin (A) and a schematic model of the Tm-Tn complex (B). F-actin was constructed with two monomers using the atomic coordinates of Lorenz et al. (1993). The globular part of Tn is located at Cys-190 of Tm (150-180) and the elongated part of TnT extends to the N-terminal region of the next Tm molecule. 


\section{2-2 FRET between Tm and Actin}

FRET between probes attached to Cys-190 of Tm and Cys-374 of actin showed almost no change in the transfer efficiency in response to a $\mathrm{Ca}^{2+}$-induced structural change of the thin filament, indicating that Tm does not change its position relative to the actin filament (Miki \& Mihashi, 1979; Lin \& Dowben, 1983; Tao et al., 1983). However, Tao et al. (1983) proposed a case where a Tm-bound donor and actin-bound acceptors can be positioned so that a movement of Tm in the grooves of F-actin produces no net change in the FRET efficiency. Acceptor probes were selectively attached to several specific points on actin (Cys-374, Lys-61, Gln-41 and the nucleotide-binding site), and a donor probe was selectively attached to Tm at positions 87 or 190 .

FRET between the probes on actin and Tm showed there was no significant change in transfer efficiency upon the addition of $\mathrm{Ca}^{2+}$ (Miki, 1990; Miki et al., 1998). Since measurement of more than three distances between points on Tm and actin uniquely position Tm on the actin filament, we can exclude the case where the movement of Tm relative to actin produces no net change in FRET efficiency. FRET also showed that the S1-induced structural change in thin filaments did not accompany Tm movement relative to the actin filament (Miki et al, 1998). In accord with these FRET measurements, polarized fluorescence measurements have suggested there is little or no changes in the position of $\mathrm{Tm}$ relative to actin upon binding of $\mathrm{Ca}^{2+}$ or upon additional binding of S1 using "ghost" fibers (Borovikov et al., 1993). On the other hand, analysis of frequency-domain FRET between Cys-190 of Tm and the phalloidin-binding site of actin (Bacchiocchi \& Lehrer, 2001) suggests that significant movement of Tm relative to actin produces no appreciable change in the FRET efficiency when the two donor positions on $\mathrm{Tm}$ in the homodimer structure of Tm are taken into account. Finding these two positions of $\mathrm{Tm}$ on actin $\left( \pm \mathrm{Ca}^{2+}\right)$ requires that the two donor probes project in definitive directions from the Tm molecule on the actin filament. Accordingly, a donor probe was selectively attached to residues 237, 245, 247, or 252 of single-cysteine Tm mutants (Miki et al., 2004). FRET between these points on Tm and Cys-374 or Gln-41 of actin did not reveal any appreciable change in the energy transfer efficiency upon binding of $\mathrm{Ca}^{2+}$ or when $\mathrm{S} 1$ binds to regulated actin (Table 1).

Table 1: Distances between probes attached to $\alpha \mathrm{Tm}$ and actin in reconstituted thin filament in the presence and absence of $\mathrm{Ca}^{2+}$ ions. Data are from Miki et al. (2004).

\begin{tabular}{|c|c|c|c|}
\hline Acceptor (actin) & Donor $(\mathrm{Tm})$ & Efficiency $(+\mathrm{Ca} / \mathrm{Ca})$ & $\mathrm{R}(2 / 3)(\AA)(+\mathrm{Ca} /-\mathrm{Ca})$ \\
\hline Cys-374 & 252 & $0.54 \pm 0.02 / 0.51 \pm 0.03$ & $38.2 \pm 0.5 / 38.9 \pm 0.8$ \\
& 247 & $0.58 \pm 0.03 / 0.58 \pm 0.02$ & $35.7 \pm 0.7 / 35.7 \pm 0.5$ \\
& 245 & $0.57 \pm 0.01 / 0.58 \pm 0.02$ & $36.1 \pm 0.2 / 35.8 \pm 0.5$ \\
& 237 & $0.68 \pm 0.01 / 0.65 \pm 0.02$ & $33.9 \pm 0.3 / 34.6 \pm 0.5$ \\
\hline Gln-41 & 252 & $0.73 \pm 0.03 / 0.62 \pm 0.04$ & $38.7 \pm 1.0 / 42.1 \pm 1.2$ \\
& 247 & $0.75 \pm 0.05 / 0.75 \pm 0.05$ & $36.6 \pm 1.7 / 36.6 \pm 1.7$ \\
& 245 & $0.76 \pm 0.05 / 0.73 \pm 0.02$ & $36.3 \pm 1.8 / 37.3 \pm 0.6$ \\
& 237 & $0.88 \pm 0.01 / 0.85 \pm 0.01$ & $32.0 \pm 0.5 / 33.4 \pm 0.4$ \\
\hline
\end{tabular}


Residues 87, 190, 237, 245, 247, and 252 of Tm are located at positions c, a, f, g, b, and $\mathrm{g}$, respectively along this seven repeat $\alpha$-helical coiled-coil structure. Donor probes at these sites project at different angles to each other on the Tm surface. Therefore we can exclude the case where movement of Tm does not accompany a net change in the transfer efficiency. An alternative and simpler analysis of the FRET data by Bacchiocci and Lehrer (2001) suggests that Tm does not change its position on actin because there is only a small change in the transfer efficiency in $\mathrm{Ca}^{2+}$ regulated actin.

\subsection{FRET between Tn and Actin}

FRET between probes attached to Cys-133 of TnI and Lys-61 or Cys-374 of actin showed that $\mathrm{TnI}$ changes its position on $\mathrm{F}$-actin when $\mathrm{Ca}^{2+}$ binds to the regulated thin filament (Miki, 1990; Tao et al., 1990). Later, using more labeling sites on TnI (Cys-9, Cys-133), TnC (Cys-98) and actin (Gln-41, Lys-61, Cys-374, and the nucleotide binding site), FRET showed that the C-terminal and inhibitory domains of TnI move towards the outer domain of actin something like a lever arm, and that the C-terminal region of $\mathrm{TnC}$ is located at the "hinge" region during this inhibition (Miki et al., 1998a).

According to the three-state model (relaxed, closed and open), $\mathrm{Ca}^{2+}$ regulates the equilibrium between the relaxed and closed states and the strong S1 binding stabilizes the open state. FRET showed that rigor S1 binding induces a positional change of TnI on F-actin (Kobayashi et al., 2001; Hai et al., 2002). The deletion mutant Tm (D234) in which internal actin-binding pseudo-repeats 2,3 , and 4 are missing, retains its ability to bind to actin and $\mathrm{Tn}$, but it inhibits actomyosin $\mathrm{Mg}$ ATPase and filament sliding in vitro, which is not reversed by $\mathrm{Ca}^{2+}$ and Tn (Landis et al., 1997). Using this functionally deficient Tm, FRET has demonstrated that the positional change of TnI corresponds well to the three states of the thin filament (Hai et al., 2002). On thin filaments reconstituted with D234Tm, TnI movement from the closed to open states was impaired, although it was normal from the relaxed to closed states. Plots of the extent of S1-induced conformational change vs. molar ratio of S1 to actin showed that the curve is hyperbolic

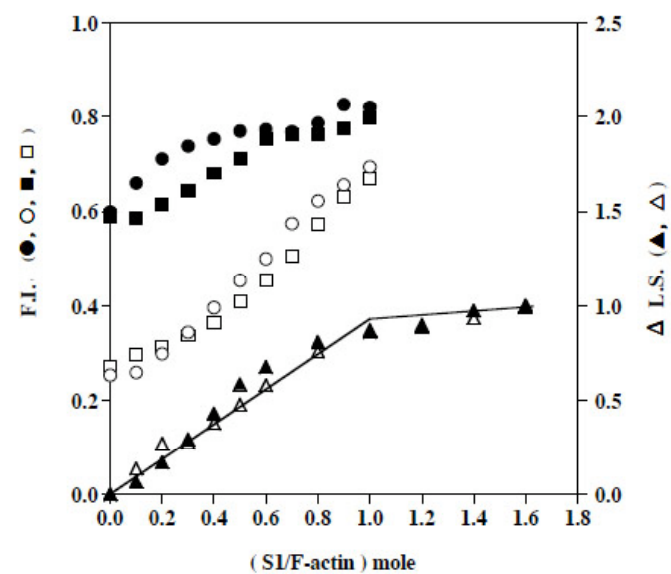

Figure 2. Relative Fluorescence Intensities (F.I.) of AEDANS bound to Cys-133 of TnI in the reconstituted thin filaments with $\mathrm{Tm}\left(\mathrm{O}\right.$ for $+\mathrm{Ca}^{2+}$ and $\bigcirc$ for $-\mathrm{Ca}^{2+}$ state) or D234eTm ( $\mathbf{a}$ for + $\mathrm{Ca}^{2+}$ and $\square$ for $-\mathrm{Ca}^{2+}$ state) vs. molar ratios of $\mathrm{S} 1$ to actin. The binding of $\mathrm{S} 1$ to the thin filament was monitored as light scattering intensity (L.S.) of the thin filament reconstituted with $\operatorname{Tm}(\boldsymbol{\Delta})$ or D234Tm $(\triangle)$ vs. molar ratios of $\mathrm{S} 1$ to actin. This figure is from Hai et al. (2002). 
in the presence of $\mathrm{Ca}^{2+}$ but sigmoidal in the absence of $\mathrm{Ca}^{2+}$. When the thin filament was reconstituted with $\mathrm{D} 234 \mathrm{Tm}$, the curve was sigmoidal whether or not $\mathrm{Ca}^{2+}$ was present (Figure 2).

$\mathrm{Ca}^{2+}$ - and $\mathrm{S} 1$-induced movements of $\mathrm{TnT}$ on the reconstituted thin filament were also detected. FRET between probes attached to residues 60 or 250 of TnT and Cys-374 or Gln-41 of actin showed significant $\mathrm{Ca}^{2+}$-dependent and $\mathrm{S} 1$-dependent changes in the position of TnT on actin (Kimura et al., 2002a). When $\mathrm{Ca}^{2+}$ binds to regulated thin filaments, TnT moves away from the outer domain of actin as seen in the case of TnI. Plots of the extent of S1-induced conformational change vs. molar ratio of S1 to actin showed that the curve is hyperbolic in the presence of $\mathrm{Ca}^{2+}$ but sigmoidal in the absence of $\mathrm{Ca}^{2+}$. However, on the thin filament reconstituted with D234Tm, the N-terminal domain of TnT did not change position upon $\mathrm{Ca}^{2+}$ binding, and the $\mathrm{C}$-terminal domain exhibited only a small movement. Plots of the extent of S1-induced conformational change vs. molar ratio of $\mathrm{S} 1$ to actin revealed that the curve is sigmoidal whether or not $\mathrm{Ca}^{2+}$ is present (Figure 3). TnT movement on the functionally deficient thin filament is strongly impaired, suggesting that it is closely related to the physiological states of the thin filament.

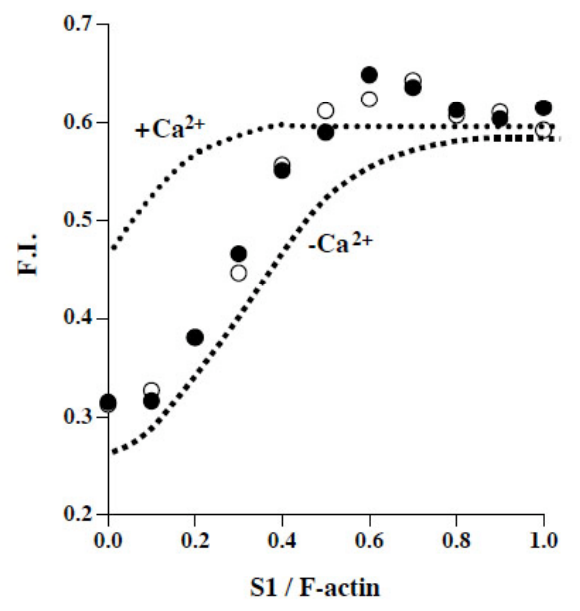

zure 3: Relative fluorescence intensities F. I. AEDANS bound to Cys-60 of TnT on thin iments reconstituted with native $\mathrm{Tm}$ (broken es) or D234Tm $\left(\bigcirc\right.$ for $+\mathrm{Ca}^{2+}$, for $\left.-\mathrm{Ca}^{2+}\right)$ molar ratios of $\mathrm{S} 1$ to actin. This figure is m Kimura et al. (2002b).

Thus far, the TnI and TnT subunits in the Tn complex change their position relative to actin corresponding to three states of the thin filament. However, when sufficient ATP is present, the S1-induced movement of $\mathrm{TnI}$ or $\mathrm{TnT}$ on the thin filament cannot be detected by FRET. This is because the population in the rigor binding state of $\mathrm{S} 1$ is extremely small in the presence of excess ATP. While, by addition of ATP under the condition where the transition curve to open state vs. molar ratios of S1 to actin shows hyperbolic, the actomyosin ATPase cycle turns actively, but under conditions where the transition curve was sigmoidal, the ATPase cycle is inhibited.

Now let us consider the meaning of the hyperbolic and sigmoidal curves. A hyperbolic transition curve means that a single S1 molecule on a unit length of actin 
filament can induce the open state. Although the population of the rigor S1 on the unit length of actin filament is very small, one S1 molecule can turn the cycle of actomyosin ATPase. The open state is one of sequential steps during the actomyosin ATPase cycle. In this case, there is no barrier for advancing to the next step. On the other hand, a sigmoidal transition curve means that cooperative binding of several S1 heads along a unit length of an actin filament is required to induce the open state. In the presence of sufficient ATP, the probability of more than two strongly bound S1s along a unit length of actin filament is very small. Thus, the system cannot pass through the open step, and consequently the actomyosin ATPase cycle does not turn on.

NEM-treated S1 binds strongly to actin even in the presence of ATP and releases the inhibition of Tm-Tn even in the absence of $\mathrm{Ca}^{2+}$ (Nagashima \& Asakura, 1982; Green et al., 1987). This phenomenon can be explained as follows. When NEM-S1 is added, the probability of several S1 molecules binding simultaneously on a unit length of actin filament becomes substantial even in the presence of ATP, enabling the ATPase cycle of the native S1 to turn actively. The three positions of Tn on thin filament correspond well to three states of thin filaments, and the transition movement of $\mathrm{Tn}$ can explain the regulation mechanism of muscle contraction.

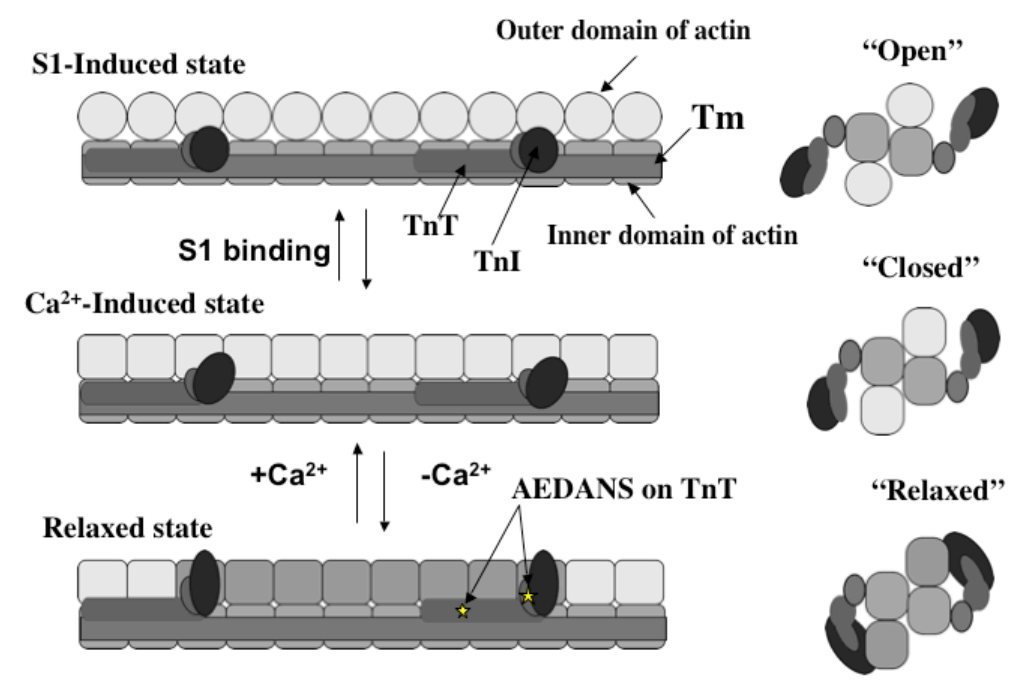

Figure 4: A schematic model of Tn and Tm along the long-pitch helix of an actin filament in three states of thin filaments (left side) and a cross section of the thin filament (right side). One actin molecule is illustrated with two frames that represent its outer and inner domains. This figure is from Kimura et al. (2002a).

Here, we illustrate the three states of thin filaments observed by FRET measurements (Figure 4). Although they clearly showed a significant extent of Tn movement relative to actin corresponding to three states of the thin filament, they did not detect any significant movement of Tm relative to actin. This model is in contrast to the steric blocking model where, intra- and/or inter-monomer flexibility of actin play an essential role in regulation. 
Instead of Tm blocking the myosin-binding site, Tn-Tm regulates this flexibility of actin filament. Cross-linking between the inner and outer domains of actin molecules by two neighboring Tn-Tm complexes along the long-pitch helix of actin filament would reduce the flexibility of the unit length of the thin filament. In an alternative model, two Tn-Tm complexes located on the opposite sides of the long-pitch helix of actin filaments control the flexibility of the thin filament.

\section{RATES OF TRANSITION BETWEEN THREE STATES OF THIN FILAMENT MEASURED BY FRET}

Using time-resolved X-ray diffraction, Kress et al. (1986) detected a rapid structural change in the thin filament before the change derived from cross-bridge movements. This structural change occurs after the stimulus with a half time of $8.5 \mathrm{~ms}$ at $14^{\circ} \mathrm{C}$ and reached a maximum in 15-20 ms. This rapid structural change after $\mathrm{Ca}^{2+}$ binding (several $\mathrm{ms}$ ) was presumed to be an azimuthal Tm movement on the actin filament. Using FRET in combination with the stopped-flow method, the rate of the TnI movement upon $\mathrm{Ca}^{2+}$ binding was determined (Miki and Iio, 1993), although no Tm movement was detected (Miki et al., 2004). The time scale of this TnI movement after $\mathrm{Ca}^{2+}$ binding was $530 \pm$ $170 \mathrm{~s}^{-1}$ at $20^{\circ} \mathrm{C}$ in good agreement with the X-ray diffraction data. Thus, it is possible that the changes in X-ray diffraction pattern interpreted as $\mathrm{Tm}$ movement are in fact caused by $\mathrm{Ca}^{2+}$-induced changes in Tn-actin interaction.

Numerous stopped-flow measurements have been carried out to study the transition rates between three states of the thin filament (Geeves and Lehrer, 1994; Maytum et al., 2002; Restar, 2002). In these experiments, fluorescent probes were attached to $\mathrm{Tm}$ or actin, and the time course of the fluorescence intensity change was traced for kinetic studies of the conformational changes of Tm and actin in response to three states of thin filaments. However, fluorescence intensities of probes on $\mathrm{Tm}$ or actin respond primarily to $\mathrm{S} 1$ binding but not to changes in $\mathrm{Ca}^{2+}$ concentration. In general, the fluorescence intensity change measured in these experiments indicates there is an environmental change around the probe, but it does not necessary mean there is a spatial rearrangement of thin filaments. On the other hand, the change in the transfer efficiency of FRET does indicate a spatial rearrangement in thin filaments. FRET between probes attached to TnI or TnT and actin on the reconstituted thin filament showed significant changes in the transfer efficiencies between three states of the thin filament. This enabled us to directly determine the transition rates between three states of the thin filament.

\subsection{The Rates of $\mathrm{Ca}^{2+}$-induced Changes of The Thin Filament}

Using FRET in combination with the stopped-flow method by rapidly changing the free $\mathrm{Ca}^{2+}$ concentration, the rate of the TnT movement between relaxed and closed states was measured as shown in Figure 5 (Shitaka et al., 2005). A FRET donor probe was attached at Cys-60 or 250 of TnT, and an acceptor was positioned at Cys-374 of actin. In both Cys-60 and 250 of TnT, the time scale of this TnT movement was $\sim 450$ and $\sim 85 \mathrm{~s}^{-1}$ at 20 ${ }^{\circ} \mathrm{C}$ on $\mathrm{Ca}^{2+}$ up and down respectively. This result correlates well with the TnI movement determined previously (Miki \& Iio, 1993). 

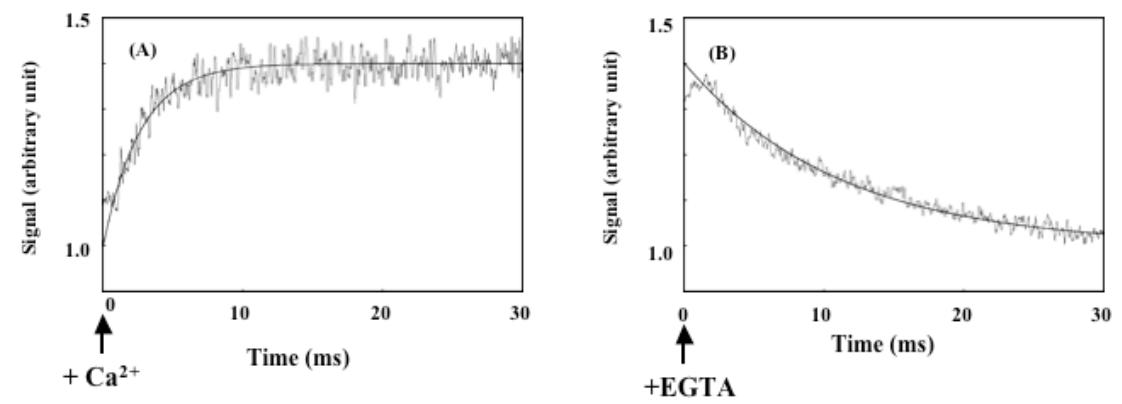

Figure 5: Stopped-flow traces of donor fluorescence intensity after rapidly changing the free $\mathrm{Ca}^{2+}$ concentrations: $\mathrm{Ca}^{2+}$ up (A) or down (B). This figure is from Shitaka et al., 2005.

\subsection{The Rates of S1-induced Changes in the Thin Filament}

After rapidly changing the concentration of rigor bound S1 by mixing the protein solutions with ATP solutions, the transition rates of TnI movement from the open to the closed or relaxed state were measured by monitoring the donor-fluorescence intensity (Shitaka et al., 2004). An energy donor probe was attached at Cys-133 of TnI, and the energy acceptor probe was attached at Cys-374 of actin. S1 binding was monitored from the scattered light under the same experimental conditions. Bound S1 was abruptly dissociated from regulated thin filaments in the presence of $\mathrm{Ca}^{2+}$ by mixing with ATP to achieve the transition from the open to closed state. The rate of dissociation increased as the concentration of ATP increased. Under these conditions S1 dissociates with a rate constant of $\sim 900 \mathrm{~s}^{-1}$ at $0.5 \mathrm{mM}$ ATP. FRET showed that TnI movement from the open to closed states occurs with a rate constant of $\sim 300 \mathrm{~s}^{-1}$ (Figure 6A). When regulated thin filaments were rapidly mixed with $\mathrm{S} 1$ in the presence of $\mathrm{Ca}^{2+}$, there is a putative a

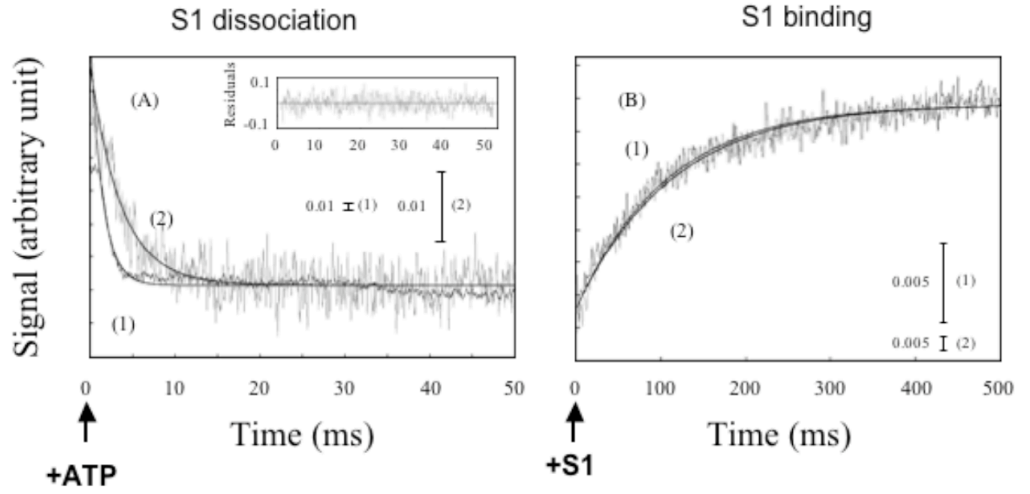

Figure 6: TnI-movement after dissociation or binding of S1 from thin filaments. Curve 1: light scattering change, Curve 2: FRET change. This figure is from Shitaka et al., 2004 
transition from the closed to open states when S1 binds. Light scattering reveals that S1 binding occurs with a rate constant of $\sim 9 \mathrm{~s}^{-1}$, and FRET showed that TnI movement occurs with almost the same rate constant as S1 binding. The result suggests that the transition from closed to open states occurs within several $\mathrm{ms}$ after S1 binding. A putative transition from the open to relaxed states occurs when bound S1 abruptly dissociates from regulated thin filaments in the absence of $\mathrm{Ca}^{2+}$. Light scattering measurements show that S1 dissociates with a rate constant of $\sim 900 \mathrm{~s}^{-1}$ while FRET shows that TnI movement occurs with two rate constants of $\sim 400 \mathrm{~s}^{-1}$ and $\sim 80 \mathrm{~s}^{-1}$. The faster rate constant $\left(k_{F L I}\right)$ is comparable to that for transition from the open to closed states observed in the presence of $\mathrm{Ca}^{2+}$. On the other hand, the slower rate constant $\left(k_{F L 2}\right)$ is similar to that for the transition from the closed to relaxed states.

These results indicate that the transition occurs in two steps, from the open to closed states, and then from the closed to relaxed state. On the other hand, when regulated thin filaments are rapidly mixed with $\mathrm{S} 1$ in the absence of $\mathrm{Ca}^{2+}$, a transition from the relaxed to open states after S1 binding has been proposed. Light scattering measurements showed that $\mathrm{S} 1$ binding occurs with a rate constant of $\sim 5 \mathrm{~s}^{-1}$. The rate constant of S1 binding to regulated thin filaments in the absence of $\mathrm{Ca}^{2+}$ is slower than that in the presence of $\mathrm{Ca}^{2+}$. The rate constants for TnI movement between three states of thin filaments induced by S1 binding or dissociation are summarized in Table 2.

Table 2: The rate constants of TnI movement between states of thin filaments induced by S1 binding/dissociation. Parentheses indicate the amplitude of the two rate constants. Data are from Shitaka et al. (2004).

\begin{tabular}{|c|c|c|c|c|c|c|c|}
\hline Donor site & \multicolumn{5}{|c|}{ S1 dissociation } & \multicolumn{2}{|c|}{ S1 binding } \\
\hline$\left(+\mathrm{Ca}^{2+}\right)$ & $\begin{array}{l}k_{\mathrm{LS}}\left(\mathrm{s}^{-1}\right) \\
\text { (L.S.) }\end{array}$ & \multicolumn{4}{|c|}{$\begin{array}{l}k_{\mathrm{FL}}\left(\mathrm{s}^{-1}\right) \\
\text { (F.I.) }\end{array}$} & $\begin{array}{l}k_{\mathrm{LS}}\left(\mathrm{s}^{-1}\right) \\
\text { (L.S.) }\end{array}$ & $\begin{array}{l}k_{\mathrm{FL}}\left(\mathrm{s}^{-1}\right) \\
\text { (F.I.) }\end{array}$ \\
\hline TnIG1C & $942 \pm 75$ & \multicolumn{4}{|c|}{$330 \pm 39$} & $8.94 \pm 1.16$ & $8.75 \pm 1.14$ \\
\hline TnICys 133 & $868 \pm 76$ & \multicolumn{4}{|c|}{$216 \pm 42$} & $9.53 \pm 1.24$ & $9.04 \pm 1.18$ \\
\hline TnIS $181 \mathrm{C}$ & $825 \pm 68$ & \multicolumn{4}{|c|}{$384 \pm 67$} & $8.91 \pm 1.16$ & $8.36 \pm 1.09$ \\
\hline$\left(-\mathrm{Ca}^{2+}\right)$ & $\begin{array}{l}k_{\mathrm{LS}}\left(\mathrm{s}^{-1}\right) \\
\text { (L.S.) }\end{array}$ & \multicolumn{2}{|c|}{$\begin{array}{c}k_{\mathrm{FL} 1}\left(\mathrm{~s}^{-1}\right) \\
\text { (F.I.) }\end{array}$} & \multicolumn{2}{|c|}{$\begin{array}{l}k_{\mathrm{FL} 2}\left(\mathrm{~s}^{-1}\right) \\
\text { (F.I.) }\end{array}$} & $\begin{array}{c}k_{\mathrm{LS}}\left(\mathrm{s}^{-1}\right) \\
(1 . \mathrm{S} .)\end{array}$ & $\begin{array}{c}k_{\mathrm{FL}}\left(\mathrm{s}^{-1}\right) \\
\text { (F.I.) }\end{array}$ \\
\hline TnIG1C & $991 \pm 89$ & $549 \pm 27$ & $(0.79)$ & $134 \pm 6.2$ & $(0.21)$ & $4.74 \pm 0.62$ & $4.52 \pm 0.59$ \\
\hline TnICys 133 & $997 \pm 83$ & $360 \pm 44$ & $(0.75)$ & $44.9 \pm 5.7$ & $(0.25)$ & $6.05 \pm 0.79$ & $4.50 \pm 0.59$ \\
\hline TnIS 181C & $836 \pm 62$ & $388 \pm 93$ & $(0.69)$ & $43.9 \pm 3.6$ & $(0.31)$ & $5.96 \pm 0.77$ & $6.09 \pm 0.79$ \\
\hline
\end{tabular}

TnT movement following dissociation or binding of S1 was measured by monitoring the donor-fluorescence intensity and light scattering (Shitaka et al., 2005). When strongly bound $\mathrm{S} 1$ is abruptly dissociated from the thin filament by mixing with ATP, the transition from the open to closed and relaxed states would occur in the presence and absence of $\mathrm{Ca}^{2+}$, respectively. A change in the light-scattering intensity indicates the dissociation of S1 from the regulated thin filament and the rate of dissociation increases as the concentration of ATP increased. At $0.5 \mathrm{mM} \mathrm{ATP,} \mathrm{it} \mathrm{was}>800 \mathrm{~s}^{-1}$. The change in the fluorescence intensity ( $\mathrm{TnT}$ movement) was analyzed by a single-exponential curve with a rate constant of $\sim 400 \mathrm{~s}^{-1}$ both in the presence and absence of $\mathrm{Ca}^{2+}$. These data indicate that the transition of TnT from the open to relaxed states occurs directly in contrast to the movement of TnI through an intermediate state (the closed state). That is 
TnT moves differentially with TnI. This differential movement suggests there is a flexible "joint" between TnI and TnT subunits in the Tn complex.

When S1 binds to the thin filament, the transition to the open state from the closed and relaxed states occurs in the presence and absence of $\mathrm{Ca}^{2+}$, respectively. Light-scattering measurements have shown that the S1 binding occurs with the rate constant of $\sim 10$ and $\sim 5 \mathrm{~s}^{-1}$ in the presence and absence of $\mathrm{Ca}^{2+}$, respectively. The rate constants for the transitions between three states of thin filaments determined by stopped-flow measurements are summarized in Table 3.

Table 3: Comparison of the rate constants of S1-induced movement of TnT. Data are from Shitaka et al. (2005)

\begin{tabular}{ccccc}
\hline $\begin{array}{c}\text { Donor Site } \\
(\mathrm{TnT})\end{array}$ & \multicolumn{2}{c}{$\mathrm{S} 1$ dissociation } & \multicolumn{2}{c}{ S1 binding } \\
\hline$\left(+\mathrm{Ca}^{2+}\right)$ & $k_{\mathrm{LS}}\left(\mathrm{s}^{-1}\right)$ & $k_{\mathrm{FL}}\left(\mathrm{s}^{-1}\right)$ & $k_{\mathrm{LS}}\left(\mathrm{s}^{-1}\right)$ & $k_{\mathrm{FL}}\left(\mathrm{s}^{-1}\right)$ \\
& $(\mathrm{L} . \mathrm{S})$. & $(\mathrm{F} . \mathrm{I})$. & $(\mathrm{L} . \mathrm{S})$. & $(\mathrm{F} . \mathrm{I})$. \\
\cline { 2 - 5 } TnT E60C & $975 \pm 107$ & $372 \pm 48$ & $10.3 \pm 1.3$ & $12.2 \pm 1.6$ \\
TnT S250C & $939 \pm 103$ & $391 \pm 51$ & $11.1 \pm 1.3$ & $11.1 \pm 1.4$ \\
\hline$\left(-\mathrm{Ca}^{2+}\right)$ & & & & \\
TnT E60C & $888 \pm 98$ & $407 \pm 53$ & $4.5 \pm 0.6$ & $5.9 \pm 0.7$ \\
TnT S250C & $828 \pm 98$ & $440 \pm 57$ & $5.7 \pm 0.7$ & $4.9 \pm 0.6$ \\
\hline
\end{tabular}

\section{CONCLUSIONS}

The structural changes of the thin filament between three states and their transition rates are illustrated in Figure 7. FRET has demonstrated that Tn has three positions on thin filaments corresponding to three states of the thin filament. However, FRET does not detect movement of Tm on thin filaments. The transition rates of Tn movement between three states are sufficient to explain the movements involved in the regulation mechanism of muscle contraction. Tn movement appears to be critical for the regulation of muscle contraction. Rather than physically blocking the myosin-binding site on the actin filament, Tm appears to regulate the flexibility of the thin and thus controls the regulation of actin-myosin interaction.
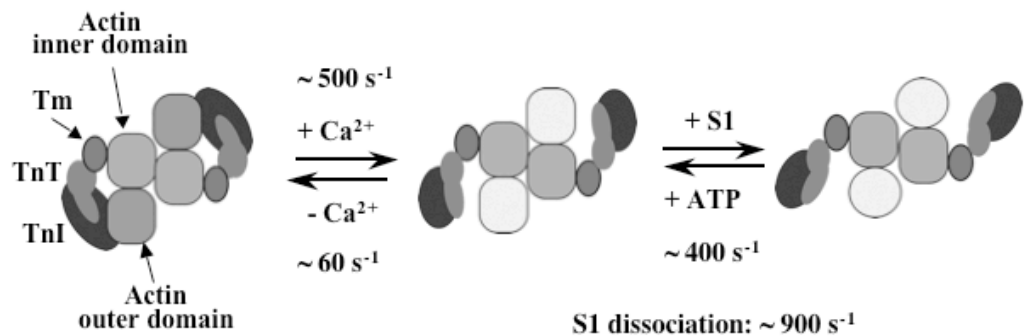

Figure 7: The rates of the transition between three states of thin filaments determined from Time-resolved FRET measurements 
Acknowledgements. The author thanks many colleagues for their helpful comments and discussion including: Drs. Y. Maéda, K. Maeda, K. Sano, T. Iio, C. Kimura, H. Hai, and Y. Shitaka. The author also thanks Dr. C. dos Remedios for critical reading of this manuscript. This work was supported in part by grant from the Special Coordination Funds of the Ministry of Education, Culture, Sports, Science and Technology, the Japanese Government.

\section{REFERENCES}

Bacchiocchi, C. and Lehrer, S.S., 2001, $\mathrm{Ca}^{2+}$-induced movement of tropomyosin in skeletal muscle thin filaments observed by multi-site FRET. Biophys. J. 82, 1524-1536.

Borovikov, Y.S, Nowak, E., Khoroshev, M.I., and Dabrowska, R., 1993, The effect of $\mathrm{Ca}^{2+}$ on the conformation of tropomyosin and actin in regulated actin filaments with or without bound myosin subfragment 1 . Biochim Biophys Acta. 1163, 280-286.

Ebashi, S., Endo, M., and Ohtsuki, I., 1969, Control of muscle contraction, Q. Rev. Biophys. 2, 351-384.

Geeves, M.A. and Lehrer, S.S., 1994, Dynamics of the muscle thin filament regulatory switch: the size of the cooperative unit. Biophys. J. 67, 273-282.

Greene, L.E., Williams, D.L.Jr., and Eisenberg, E., 1987, Regulation of actomyosin ATPase activity by troponin-tropomyosin: Effect of the binding of the myosin subfragment 1 (S-1), ATP complex. Proc. Natl. Acad. Sci. USA 84, 3102-3106.

Hai, H., Sano, K., Maeda, K., Maéda Y., and Miki, M., 2002, $\mathrm{Ca}^{2+}$-induced conformational change of reconstituted skeletal muscle thin filaments with an internal deletion mutant d234-tropomyosin observed by fluorescence energy transfer spectroscopy: Structural evidence for three states of thin filament. $J$. Biochem. 131, 407-418.

Haselgrove, J., 1972, X-ray evidence for a conformational change in the actin containing filaments of vertebrate striated muscle. Cold Spring Harbor Symp. Quant. Biol. 37, 341-352.

Holmes, K.C., 1995, The actomyosin interaction and its control by tropomyosin. Biophys. J. 68, 2s-7s.

Huxley, H.E., 1972, Structural changes in the actin- and myosin-containing filaments during contraction. Cold Spring Harb. Symp. Quant. Biol. 37, 361-376.

Kimura, C., Maeda, K., Maéda, Y., and Miki, M., 2002a, $\mathrm{Ca}^{2+}$ - and S1-induced movement of troponin T on reconstituted skeletal muscle thin filaments observed by fluorescence energy transfer spectroscopy. J. Biochem. 132 93-102.

Kimura, C., Maeda, K., Hai, H., and Miki, M., 2002b, $\mathrm{Ca}^{2+}$ - and S1-induced movement of troponin T on mutant thin filaments reconstituted with functionally deficient mutant tropomyosin. J. Biochem. 132, 345-352.

Kobayashi, T., Kobayashi, M., and Collins, J.H., 2001, $\mathrm{Ca}^{2+}$-dependent, myosin subfragment 1-induced proximity changes between actin and the inhibitory region of troponin I. Biochim. Biophys. Acta 1549, 148-154.

Kress, M., Huxley, H.E., Faruqi, A.R., and Hendrix, J., 1986, Structural changes during activation of frog muscle studied by time-resolved x-ray diffraction, J. Mol. Biol. 188, 325-342.

Landis, C.A., Bobkova, A., Homsher, E., and Tobacman, L.S., 1997, The active state of the thin filament is destabilized by an internal deletion in tropomyosin. J. Biol. Chem. 272, 14051-14056.

Lin, T-I., and Dowben, R.M., 1983, Studies on the spatial arrangement of muscle thin filament proteins using fluorescence energy transfer. J. Biol. Chem. 258, 5142-5150.

Lorenz, M., Popp, D., and Holmes, K.C., 1993, Refinement of the F-actin model against x-ray fiber diffraction data by the use of a directed mutation algorithm. J. Mol. Biol. 234, 826-836.

Maytum, R., Geeves M.A., and Lehrer S.S., 2002, A modulatory role for the troponin T tail domain in thin filament regulation. J. Biol. Chem. 277, 29774-29780.

McKillop, D. F. and Geeves, M.A., 1993, Regulation of the interaction between actin and myosin subfragment 1: evidence for three states of the thin filament. Biophys. J. 65, 693-701.

Miki, M. and Mihashi, K., 1979, Conformational change of the reconstituted thin filament - fluorescence energy transfer and fluorescence polarization measurements. Seibutsu-Butsuri 19, 135-140 (in Japanese).

Miki, M., 1990, Resonance energy transfer between points in a reconstituted skeletal muscle thin filament : A conformational change of the thin filament in response to a change in $\mathrm{Ca}^{2+}$ concentration. Eur. J. Biochem. 187, 155-162.

Miki, M. and Iio, T., 1993, Kinetics of structural changes of reconstituted skeletal muscle thin filaments observed by fluorescence resonance energy transfer. J. Biol. Chem. 268, 7101-7106. 
Miki, M., Kobayashi, T., Kimura, H., Hagiwara, A., Hai, H., and Maéda, Y., 1998, $\mathrm{Ca}^{2+}$-induced distance change between points on actin and troponin in skeletal muscle thin filaments estimated by fluorescence resonance energy transfer spectroscopy. J. Biochem. 123, 324-331.

Miki, M., Miura, T., Sano, K., Kimura, H., Kondo, H., Ishida, H., and Maéda, Y., 1998, Fluorescence resonance energy transfer between points on tropomyosin and actin in skeletal muscle thin filaments: Does tropomyosin move? J. Biochem. 123, 1104-1111.

Monteiro, P.B., Lataro, C., Ferro, J.A., and Reinach, F.C., 1994, Functional a-tropomyosin produced in Escherichia coli. J. Biol. Chem. 269, 10461-10466.

Nagashima, H., and Asakura, S., 1982, Studies on co-operative properties of tropomyosin-actin and tropomyosin-troponin-actin complexes by the use of N-ethylmaleimide-treated and untreated species of myosin subfragment 1. J. Mol. Biol. 155, 409-428.

Narita, A., Yasunaga, T., Ishikawa, T., Mayanagi, K., and Wakabayashi, T., 2001, $\mathrm{Ca}^{2+}$-induced switching of troponin and tropomyosin on actin filaments as revealed by electron cryo-microscopy. J. Mol. Biol. 308, 241-261.

Ohtsuki, I., Maruyama, K., and Ebashi, S., 1986, Regulatory and cytoskeletal proteins of vertebrate skeletal muscle, Adv. Protein Chem. 38, 1-67.

Resetar, A.M., Stephens, J.M., and Chalovich, J.M., 2002, Troponin-tropomyosin: An allosteric switch or a steric blocker? Biophys. J. 83, 1039-1049.

Squire, J.M. and Morris, E.P., 1998, A new look at thin filament regulation in vertebrate skeletal muscle. FASEB J. 12, 761-771.

Shitaka, Y., Kimura, C., Iio, T., and Miki, M., 2004, Kinetics of the structural transition of muscle thin filaments observed by fluorescence resonance energy transfer. Biochemistry 46, 10739-10747.

Shitaka, Y., Kimura, C., and Miki, M., 2005, The rates of switching movement of troponin-T between three states of skeletal muscle thin filaments determined by fluorescence resonance energy transfer. J. Biol. Chem. 280, 2613-2619.

Tao, T., Gong, B.J. and Leavis, P.C., 1990, Calcium-induced movement of troponin-I relative to actin in skeletal muscle thin filaments. Science 247, 1339-1341.

Tao, T., Lamkin, M., and Lehrer, S.S., 1983, Excitation energy transfer studies of the proximity between tropomyosin and actin in reconstituted skeletal muscle thin filaments. Biochemistry 22, 3059-3066.

Vibert, P., Craig, R., and Lehman, W., 1997, Steric-model for activation of muscle thin filaments. J. Mol. Biol. 266, 8-14.

White, S.P., Cohen, C., and Phillips Jr, G.N., 1987, Structure of co-crystals of tropomyosin and troponin. Nature 325, 826-828. 